HNO 2011 · 59:951-952

DOI 10.1007/s00106-011-2356-2

Online publiziert: 20. Juli 2011

(c) Springer-Verlag 2011

\author{
K.-B. Hüttenbrink \\ Klinik und Poliklinik für Hals-Nasen-Ohrenheilkunde, Kopf- und Halschirurgie, \\ Universitätsklinikum Köln
}

\section{Mittelohrmechanik und Mittelohrchirurgie} Operative und technische Entwicklungen
Als sich in der Mitte des 19. Jh. die naturwissenschaftliche Medizin entwickelte, wagten sich erste Chirurgen auch in das Mittelohr zur Behandlung der damals lebensbedrohlichen Infektionen. Sie verwendeten Meißeltechniken und legten große Höhlen zum Eiterabfluss an, ohne Rücksicht auf die Funktion des Ohrs nehmen zu können.

Erst Mitte des vorigen Jahrhunderts trat durch die Einführung des binokularen Op.-Mikroskops die Ohrchirurgie in eine neue Ära. Erstmals war es möglich, nicht nur gesichtsnerverhaltend, sondern sogar funktionsverbessernd zu operieren. $\mathrm{Zu}$ den segensreichen chirurgischen Entwicklungen zählen die Rekonstruktion des Trommelfells, der Wiederaufbau der zerstörten Gehörknöchelchenkette und nach Dekaden fast dogmatischer „Glaubenskriege“ über offene oder geschlossene Techniken - die Anwendung individualisierter Eradikationsverfahren. Mit der Wiederherstellung der biologischen Funktion und der Feinmechanik des Mittelohrs erlangen Patienten so auch nach ausgedehnten zerstörerischen Krankheitsprozessen in vielen Fällen wieder ein soziales Gehör.

\section{( Manchen Patienten bleibt ein Hörerfolg verwehrt, wenn die Belüftung der Paukenhöhle fehlt}

Trotz dieser operativen und technischen Fortschritte bleibt jedoch manchen Patienten ein Hörerfolg verwehrt, wenn die
Belüftung der Paukenhöhle fehlt. Zwar ist seit Politzers Beschreibungen die Funktion der Tube selbst nicht - wie früher angenommen - die Ursache der zahlreichen Erkrankungen, auch nicht der des Innenohrs. Die Vorgänge innerhalb und außerhalb der Tube, ihre Bedeutung für die funktionsentscheidende Luftfüllung des Mittelohrs und die Frage, ob ihre Funktionsstörung als Ursache oder nur als Folge der chronischen Mittelohrentzündungen zu bewerten ist, konnte trotz aller Forschungsanstrengungen seit über 100 Jahren nicht abschließend geklärt werden. Widersprüchliche Ergebnisse unzähliger Versuchsaufbauten zur Bedeutung der Tubenbelüftung mit Interpretationen unphysiologischer Tubenöffnungsversuche bis hin zu Selbstversuchen heroischer Ohrforscher haben nicht schlüssig beweisen können, ob die Regulation des Luftgehalts im Mittelohr mit einer wie auch immer gearteten Tubendurchgängigkeit zusammenhängt oder ob die Tube vielleicht nur ein Drainagesystem für die Schleimhautauskleidung des Mittelohrs darstellt, in Analogie zu allen mit Schleimhaut ausgekleideten Höhlen des Körpers. Einige Hinweise auf die Bedeutung der Mittelohrschleimhaut für die Gasproduktion und Resorption weisen in diese Richtung einer Reinigungsfunktion der Tube.

Eine kausale Therapie bei permanent gestörten Belüftungsverhältnissen, die entscheidend die Funktionsfähigkeit des auf einem Luftpolster schwingenden Trommelfell-Gehörknöchelchen-Apparats beeinflussen, erweist sich häufig als nicht er- folgreich, da wir den entscheidenden $\mathrm{Pa}$ thomechanismus zur Belüftungsregulation des Mittelohrs noch nicht kennen.

Umso erfolgreicher ist in den letzten Jahrzehnten die rekonstruktive Funktionschirurgie des Mittelohrs unter der Voraussetzung guter Belüftungsverhältnisse geworden. Nach Wullsteins wegweisenden Untersuchungen entwickelte sie sich rasant mit dem Hauptaugenmerk auf die Verwendung geeigneter Materialien zum Ersatz einer defekten Gehörknöchelchenkette bis zu den modernen Titanimplantaten. Die Formgebung und damit die akustische Qualität der Mittelohrprothesen blieb über lange Zeit im Bereich empirischer Erfahrung. Erst die Einführung der Laser-Doppler-Vibrometrie brachte genauere Einblicke in die Schwingungsform und die Funktion der intakten und der rekonstruierten Ossikelkette. Zum Verständnis der komplexen Mechanik der vibrierenden Strukturen ist die Ohrchirurgie auf die wissenschaftliche Zusammenarbeit mit den Ingenieurswissenschaften angewiesen, was sich auch in den Beiträgen zu diesem Thema auf internationalen Kongressen zeigt. Neue Entwicklungen im Design wie in der Wahl der Materialien haben zu einer Vielzahl neuer Mittelohrprothesen geführt. Sie versprechen einen akustisch effizienten und gleichzeitig stabilen Schalltransport durch das Mittelohr unter Berücksichtigung der hydraulischen Verstärkerfunktion.

Ein ganz neues Kapitel wurde in den letzten Jahren mit der Einführung implantierbarer Hörgeräte in die Mittel- 
ohrchirurgie aufgeschlagen. Der Traum vieler Ohrchirurgen und Ingenieure bestand darin, von der passiven Schwingungsfähigkeit des Trommelfells als Motor der Mittelohrübertragung unabhängig zu sein. Dies gelang mit zunehmender Miniaturisierung und Weiterentwicklung der Implantate und führte zum erstmals möglichen routinemäßigen Einsatz implantierbarer Systeme. Eigentlich entwickelt für die Behandlung sensorineuraler Schwerhörigkeiten zur direkten Anregung der Cochlea unter Umgehung des Mittelohrapparats, lassen sich diese Minivibratoren problemlos auch bei völlig zerstörten Mittelohrverhältnissen anwenden. Die Einbindung der Ingenieurswissenschaft in die Ohrchirurgie legt den Vergleich nahe zur Technikgeschichte des Fliegens mit motorgetriebenen Flugzeugen: Die Funktion wird unabhängig von der Natur bzw. der biologischen Funktion (des Mittelohrs). In der Weiterentwicklung der Techniken wird die Implementierung modernster signalverarbeitender Verfahren in die Hörgeräte zeigen, dass wir am Anfang einer für unsere Patienten vielversprechenden Entwicklung stehen. Selbst in aussichtslosen Fällen eines desolaten Mittelohrs wird es möglich, dem Patienten ein soziales Gehör zurückzugeben.

\section{( ) Minivibratoren lassen sich auch bei völlig zerstörten Mittel- ohrverhältnissen anwenden}

Eine derartig optimistische Prognose war bisher aufgrund der Unvorhersehbarkeit postoperativer Belüftung und Funktionsfähigkeit des Mittelohrs nicht möglich.

Die Fortschritte in der rekonstruktiven Mittelohrchirurgie dürfen jedoch nicht vergessen lassen, dass eine Op. stets einen blutigen und $z$. B. im chronisch entzündeten Gebiet diffizilen Eingriff bedeutet. Aufgrund der engen Nachbarschaftsbeziehungen zu vulnerablen und vitalen Strukturen stellt das Mittelohr - eingeklemmt zwischen Dura und großen Blutleitern, quer durchzogen vom Gesichtsnerv, trotz oder gerade wegen seiner Beschränkung auf wenige Kubikzentimeter - ein anspruchsvolles Op.-Gebiet dar. Erst nach einer langen Trainingsphase besitzt der angehende Ohrchirurg genügend Erfahrung, die dreidimensionalen Struktu- ren im stereomikroskopischem Blick korrekt einzuordnen und die Beseitigung der Krankheitsursachen mit der folgenden Rekonstruktion der Mikromechanik erfolgreich abzuschließen. Auch der erfahrene Ohrchirurg ist nie vor Komplikationen gefeit. Entscheidend bleibt dabei die rechtzeitige Erkennung gefahrenträchtiger Konstellationen, auch postoperativ.

Von grundlegender Wichtigkeit für die Ausbildung des Ohrchirurgen ist, seine Erfahrungen in der Mittelohrchirurgie durch das Üben an natürlichen Felsenbeinen zu gewinnen. Leider bieten sich dazu immer weniger Möglichkeiten. Industriell hergestellte Felsenbeine stellen hier nur einen unzureichenden Ersatz dar. Aus diesem Grund muss die Erfahrung in der Op. am Patienten gewonnen werden, was eine personalaufwendige, vom betriebswirtschaftlich organisierten System der DRGMedizin nicht berücksichtigte Überwachung impliziert. Dies mag zusammen mit den zunehmenden haftpflichtbelasteten Erfolgsversprechen der Chirurgie die Ursache dafür sein, dass mancherorts bereits dem Hörgerät der Vorzug gegeben wird gegenüber der hörverbessernden Ohr-Op. Eine solche Entwicklung, in der die Potenziale erfolgreicher Mittelohrchirurgie nicht ausgeschöpft werden, vergibt die Chance, über die Wiederherstellung des sozialen Gehörs die Lebensqualität der Patienten entscheidend zu verbessern.
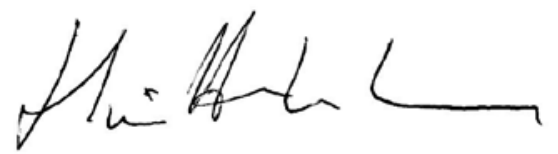

Karl-Bernd Hüttenbrink

\section{Korrespondenzadresse}

\section{Prof. Dr. Dr. h.c. K.-B. Hüttenbrink}

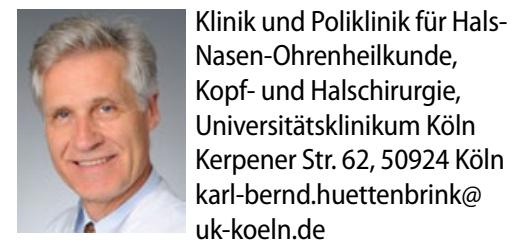

\section{Neuer Therapieansatz für Usher-Syndrom}

Das Usher-Syndrom ist mit einer Häufigkeit von 1:6000 die häufigste Form angeborener Taub-Blindheit des Menschen. Wissenschaftler der Universität Mainz haben nun einen neuen Therapieansatz für die Krankheit nachgewiesen.

Bei der Evaluation potenzieller Therapiemöglichkeiten liegt der Fokus der Forscher auf einer Mutation im USH1C-Gen, die zu einer schweren Form des Usher-Syndroms führt. Bei dieser Nonsense-Mutation im USH1C-Gen entsteht ein Stopp-Signal in der DNA und die Proteinsynthese wird vorzeitig abgebrochen.

Die Wissenschaftler konnten zeigen, dass das Molekül PTC124 (Ataluren ${ }^{\circledast}$ ) das Überlesen des Stopp-Signals im mutierten USH1C-Gen auslöst, die Proteinsynthese weiterläuft und das funktionelle Genprodukt in den Zellen hergestellt wird. Der Wirkstoff PTC124 zeigte in der Studie darüber hinaus eine hohe Verträglichkeit in Netzhautkulturen der Maus und des Menschen. Zudem gelang es dem Team erstmals, das Überlesen einer Mutation im Auge in vivo nachzuweisen.

Neben weiterführenden präklinischen Untersuchungen zur Anwendung der Wirkstoffe im Auge plant das Mainzer Usher-Labor, das neuartige Verfahren zur Therapie des Usher-Syndroms möglichst zeitnah in die Klinik direkt zum Patienten zu bringen.

Literatur: Goldmann T, Overlack N, Wolfrum U et al (2011) PTC124-mediated translational readthrough of a nonsense mutation causing Usher syndrome type 1C. Hum Gene Ther 22(5):537-47

Quelle: Johannes Gutenberg-Universität Mainz, www.uni-mainz.de 